\title{
Ecology of Bloodstream Infections and Temporal Trends of Their Antibiograms with Respect to Source and Duration of Incubation: A 5-Year Retrospective Observational Analysis
}

\author{
Amit Banik, Valarie W. Lyngdoh² Elantamilan Durairaj ${ }^{2} \quad$ Anil C. Phukan² Raghavendra Kotal ${ }^{3}$
}

${ }^{1}$ All India Institute of Hygiene \& Public Health, Kolkata, West Bengal, India

2Department of Microbiology, NEIGRIHMS, Shillong, Meghalaya

${ }^{3}$ Department of Anaesthesiology \& Critical Care, NEIGRIHMS, Shillong, Meghalaya

J Lab Physicians:2020;12:56-67

\author{
Address for correspondence Amit Banik, MBBS, MD, DNB, \\ All India Institute of Hygiene \& Public Health, Room\# 203, AllH\&PH, \\ BN Campus, Kolkata, West Bengal 700106, India \\ (e-mail: dramitbanik@gmail.com).
}

\begin{abstract}
Keywords

- bloodstream infection

- Meghalaya

- antimicrobial sensitivity

- blood culture

- empirical therapy

Purpose Blood is one of the most important connective tissues of human body. Bloodstream infection can range from inapparent bacteremia till fulminant septic shock with high mortality. Presence of microbes in blood whether continuously, intermittently, or transiently is a grave risk to every organ of body. Culture of blood is a vital tool to diagnose such infections. Drug susceptibility patterns help in rationalizing therapy.

Objective The aim of the study is to perform bacteriological analysis and assess drug sensitivity patterns of blood culture isolates and compare in light of other associated variables.

Design Retrospective observational study was conducted from January 2009 to December 2013 at a tertiary care hospital at Shillong, India. Blood samples were collected with aseptic guidelines and cultured for 7 days. Growths were identified by standard biochemical tests and subjected to sensitivity testing according to Modified Kirby Bauer disk diffusion method. Data for source of blood collection and duration of incubation were noted and compared.

Results Atotal of 658(11.2\%) pathogenswere isolated from 5,867 bacteremia-suspected patient blood specimens. Contamination was observed at the rate of $1.21 \%$. Gram-negative organisms were the predominant pathogens recovered, Klebsiella pneumoniae being the most common. No significant difference was observed between the number of organisms isolated within or beyond 48 hours. Acinetobacter baumannii and $K$. pneumoniae have significantly higher chances $(p<0.05)$ of isolation from central line catheters compared with peripheral venipuncture.

Conclusion Successful treatment of sepsis depends on early diagnosis and proper antimicrobial therapy. Local knowledge of bacteriological profile and antimicrobial sensitivity patterns helps rationalize empiric treatment strategies.
\end{abstract}

\section{Introduction}

Blood is in the truest sense the elixir of life. It contains a part of the extracellular fluid along with a wide variety of other constituents which are indispensable for proper functioning and survival of human life. From providing nutrients, limiting pathogens, perfusing and ventilating organs, clotting wounds, removal of toxins and chemicals, and dissemination of hormones and drugs throughout the body it performs a pivotal role in body defense and survival. Presence of organisms in blood can give rise to different clinical scenarios. Clinical presentation ranges from benign transient bacteremia with

(C)2020 by The Indian Association of Laboratory Physicians
License terms

(®) (1) $\ominus \circledast$ 
little or no symptoms to fulminant septic shock with high mortality. ${ }^{1}$

Bacteremia or fungemia denotes the presence of viable bacteria or fungi in the blood with or without clinical symptoms. Systemic inflammatory response syndrome (SIRS) is defined by the presence of two or more acute findings (tachycardia, leukocytosis, or leukocytopenia, fever or hypothermia, tachypnea). Combination of SIRS and bacteremia is known as sepsis which is a host systemic response to infection. Sepsis with additional acute organ dysfunction due to documented or suspected infection is known as severe sepsis. Septic shock is defined as severe sepsis with hypotension which is unresponsive to fluid resuscitation. ${ }^{2}$ Blood stream infections (BSI) are a major cause of morbidity and mortality and are among the most common health care-associated infections. With an attributable mortality rate of $15 \%$, it is an eminent cause of death worldwide. ${ }^{1}$

Presence of microbes in blood whether continuously, intermittently, or transiently is a grave risk to every organ of the body. Early diagnosis plays a crucial role in managing BSI and hence prompt detection of such infections is a critical function of clinical microbiology laboratories. Blood culture is a vital tool for the detection of BSI and is the gold standard for bacteremia detection. Initial antimicrobial empirical therapy being very imperative in BSI, must be based on the knowledge of the bacterial profile and their sensitivity patterns. ${ }^{3}$ Irrational use of drugs leads to an increase of multidrug-resistant bugs and thus worsens the management of the infections. Prevalence and susceptibility patterns of microorganisms vary according to the geography and use of antibiotics in different health care settings. There is paucity of similar reports with regard to disease burden from Northeast India, and more so from Meghalaya. This region is unique with respect to its ethnicity, geographical location, topography, climatic condition unlike the rest of the country. The current study intends to report the prevalence, bacteriological analysis of microorganisms, and antimicrobial susceptibility profiles of blood culture isolates and other auxiliary variables in a tertiary health care center in Northeast India.

\section{Materials and Methods}

The present study is a 5-year retrospective observational analysis of blood culture isolates received in the Department of Microbiology of the hospital from January 2009 to December 2013. Necessary Ethics Committee Approval was obtained for the study.

\section{Sample Collection}

Blood specimens were obtained at bedside either by a trained phlebotomist or by nursing staff from wards and critical care units. The skin was disinfected with $2 \%$ chlorhexidine. Approximately 5 to $10 \mathrm{~mL}$ of blood was collected from adult patients, 1 to $5 \mathrm{~mL}$ from pediatric patients, and 1 to $2 \mathrm{~mL}$ from neonates. The antecubital and median cubital fossa were the preferred sampling sites using a needle and syringe. The blood samples from the central vein catheters were obtained from needleless caps that have been disinfected with 70\% isopropyl alcohol, allowed to dry, and wiped with sterile gauze prior to obtaining the sample.

\section{Sample Processing}

Blood for culture was collected from 5,867 clinically suspected bacteremia cases under strict aseptic precautions. They were inoculated into conventional blood culture bottles containing Brain Heart Infusion broths (1:10 dilution). These were incubated aerobically at $37^{\circ} \mathrm{C}$, observed for turbidity every morning, and manually agitated for aeration for 7 complete days. ${ }^{4}$ Regular blind subcultures were done at the completion of day 2 and day 7 of aerobic incubation. Subcultures were done on MacConkey agar, 5\% Sheep blood agar, and chocolate agar as and when turbidity was noticed. Specimens were discarded after 7 days of unsuccessful aerobic incubation. ${ }^{5}$ Any growth obtained was processed and identified by Gram staining, colony morphology, and standard biochemical tests. Antibiotic susceptibility testing was done according to Kirby Bauer disk diffusion method and interpreted according to Clinical and Laboratory Standards Institute (CLSI) guidelines. ${ }^{6}$ Positive growths were further critically analyzed based on the criteria to be agents of bacteremia, fungemia, and contaminants. ${ }^{7-9}$ The data were manually compiled and analyzed critically for the study. The following strains were used as quality control strains:

1. Staphylococcus aureus (ATCC 25923)

2. Escherichia coli (ATCC 25922)

3. Pseudomonas aeruginosa (ATCC 27853)

\section{Results}

The present study involves 5,867 continuous samples received from different wards and intensive care units (ICUs) of the hospital. Among them, 740 isolates showed positive aerobic bacterial growth and $669(11.40 \%)$ of them were recognized pathogens. However, blood bank surveillance revealed 11 pathogens and 17 contaminants. Finally, 658(11.2\%) isolates were recovered from patients as incriminating microorganisms responsible for bacteremia/fungemia. More males suffered from bacteremia than females with a gender ratio skewed at 1.4:1, (-Supplementary Table S1, online only). The mean age of patients was $33.67 \pm 23$ years (range $0-85$ years). Bulk of the specimens were sent from Medicine, Pediatric wards apart from critical care units. Data including the most common clinical syndromes leading to bacteremia/fungemia and the corresponding distribution of the microbial agents responsible are depicted in -Table 1. Cerebrovascular accidents and their subsequent complications were the leading cause for BSIs. Contamination in blood culture was documented in $71(1.21 \%)$ isolates in the present study. Most of them were from blood bank and pediatric ICU. A detailed analysis of contaminants obtained from different sections of hospital is presented in - Table 2. Among 658 isolates recovered from patients, the spectrum of microbes included 436 (66.3\%) gram-negative bacilli (GNB), 195 (29.6\%) gram-positive cocci (GPC), 15 gram-negative cocci, 1 gram-positive bacilli, 
58 Bloodstream Infection Trends in Meghalaya Banik et al.

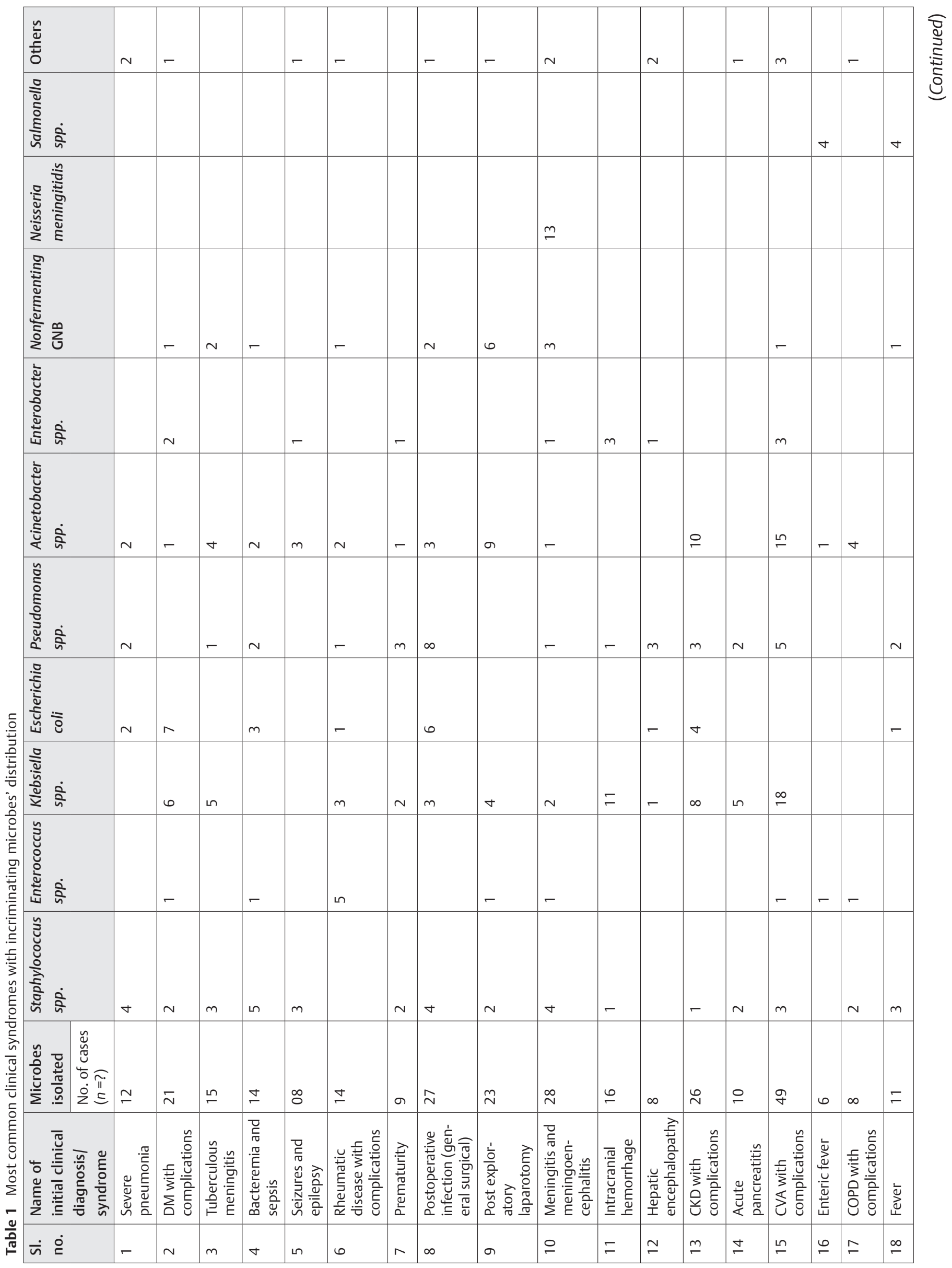

Journal of Laboratory Physicians Vol. 12 No. 1/2020 


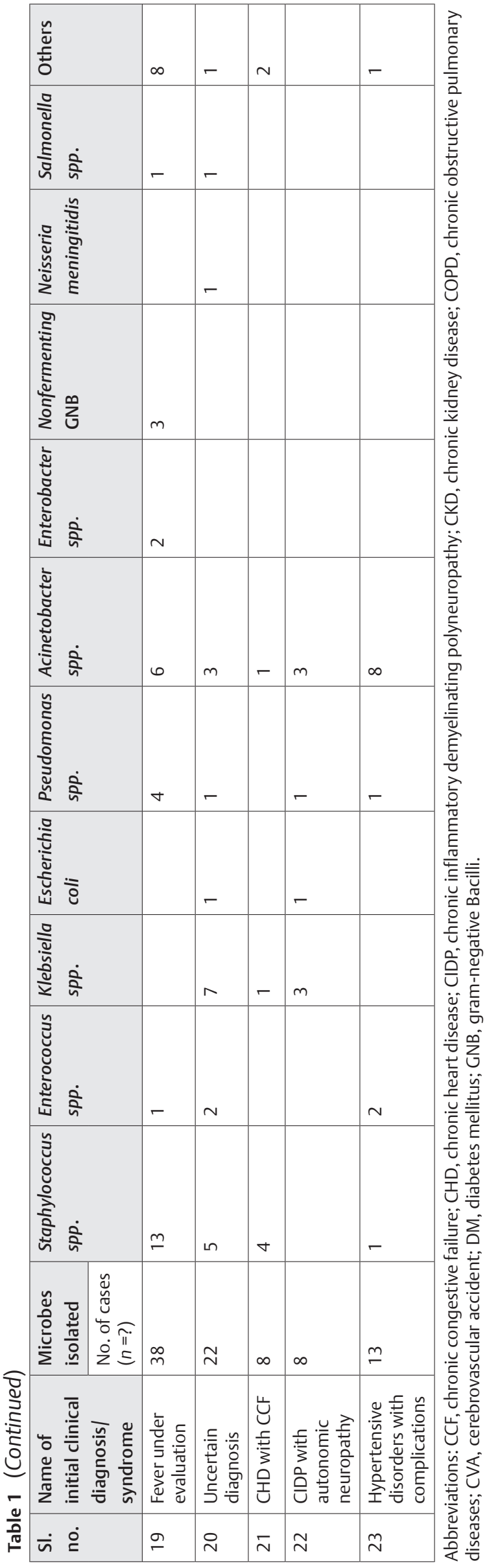

and 11 yeasts. Isolation of GNBs was significantly higher $(p<0.001)$ than other groups of organisms. Within GNBs, Klebsiella pneumoniae (28.67\%) was the dominant isolate obtained followed by Acinetobacter baumannii (22.47\%) and Pseudomonas aeruginosa (14.45\%). Eleven isolates of Salmonella spp. and one isolate of Hemophilus influenzae were also recovered. As a group, Enterobacteriaceae comprised half of all GNBs and 32.67\% of total pathogens recovered. Among GPCs isolated, S. aureus (65.6\%) was the dominant organism followed by Enterococcus spp. (15.38\%) and Streptococcus spp. (11.8\%). One-third of Enterococcus spp. isolates carried highlevel aminoglycoside resistance (HLAR) genes. Streptococcus pneumoniae was recovered from seven bacteremic patients. Of them, five were children younger than 9 years and two elderly older than 50 years. They were most likely severe cases of pneumococcal pneumonia causing bacteremia. Fifteen isolates of Neisseria meningitidis were recovered from the study. Neisseria meningitidis is a dreaded organism considering the capability of this organism to rapidly deteriorate relatively benign bacteremia into fulminant bacteremia and septic shock. Worth mentioning here is that all these cases were recovered during an outbreak between November 2008 and April 2009.

Fungemia confirmed in 11 cases were mostly Candida spp. A single case of bacteremia by Listeria monocytogenes was also detected in a 2-year-old child who presented with acute gastroenteritis. Comparison of isolation rates of organisms on different days of incubation shows that chances of isolation of microbes within the second day of incubation was significantly $(p<0.001)$ higher than other days. The distribution of different isolates and groups recovered with each passing day of incubation is presented in - Table 3. Significant isolation ( $n=242$, 36.7\%) of organisms was obtained subsequent to turbidity detection on the second day. This was followed by higher detection of organisms on the third day $(n=115)$ and first day $(n=89)$ of incubation. A total of 331 isolates were recovered after detecting turbidity within the first 48 hours of aerobic incubation compared with 327 isolates of organisms which were recovered when incubation was continued beyond 48 hours till 7 days. Interestingly, both K. pneumoniae and Escherichia coli were consistently isolated better in significant proportions ( $p<0.001$ ) within the first 48 hours. Almost 73.8 and $80 \%$ isolates of $K$. pneumoniae and $E$. coli, respectively were detected within initial 48 hours. Concurrently, organisms which have significantly higher chances of recovery after initial 48 hours include Candida spp. $(p=0.006), N$. meningitidis $(p=0.017)$, Salmonella paratyphi $(p=0.044)$, methicillin-resistant S. aureus (MRSA; $p<0.001$ ), and methicillin-sensitive S. aureus (MSSA, $p=0.024)$. However, there was no significant difference between number of organisms isolated within or beyond 48 hours till 7 days. A comprehensive distribution frequency table shows different microbes with their growth patterns at 48 hours and at 7 days (-Table 4). A comparison of data about the source of blood collection shows that $A$. baumannii and $K$. pneumoniae have significantly higher chances $(p<0.05)$ of isolation from central line catheters compared with peripheral venipuncture, while 
Table 2 Details of samples, pathogens, and contaminants from different wards

\begin{tabular}{|c|c|c|c|c|c|c|c|c|}
\hline \multirow[t]{2}{*}{ Department } & \multirow[t]{2}{*}{ Total samples } & \multirow[t]{2}{*}{$\begin{array}{l}\text { Negative culture } \\
\text { samples }\end{array}$} & \multicolumn{2}{|c|}{$\begin{array}{l}\text { Organisms } \\
\text { isolated }\end{array}$} & \multicolumn{2}{|c|}{$\begin{array}{l}\text { Pathogen } \\
\text { yield }\end{array}$} & \multicolumn{2}{|c|}{ Contamination } \\
\hline & & & & (\%) & & (\%) & & $(\%)$ \\
\hline Blood bank & 181 & 153 & 28 & 15.47 & 11 & 6.08 & 17 & 9.39 \\
\hline Cardiology & 326 & 315 & 11 & 3.37 & 10 & 3.07 & 1 & 0.31 \\
\hline Medicine & 1,236 & 1,162 & 74 & 5.99 & 64 & 5.18 & 10 & 0.81 \\
\hline CTVS & 147 & 127 & 20 & 13.61 & 19 & 12.93 & 1 & 0.68 \\
\hline Dialysis unit & 8 & 6 & 2 & 25.00 & 2 & 25.00 & 0 & 0.00 \\
\hline Otolaryngology & 16 & 16 & 0 & 0.00 & 0 & 0.00 & 0 & 0.00 \\
\hline General surgery & 40 & 38 & 2 & 5.00 & 1 & 2.50 & 1 & 2.50 \\
\hline Coronary care unit & 172 & 155 & 17 & 9.88 & 17 & 9.88 & 0 & 0.00 \\
\hline ICU & 1,170 & 800 & 370 & 31.62 & 362 & 30.94 & 8 & 0.68 \\
\hline Neurology & 60 & 59 & 1 & 1.67 & 1 & 1.67 & 0 & 0.00 \\
\hline Obstetrics and gynecology & 143 & 134 & 9 & 6.29 & 8 & 5.59 & 1 & 0.70 \\
\hline Oncology & 42 & 42 & 0 & 0.00 & 0 & 0.00 & 0 & 0.00 \\
\hline Ophthalmology & 2 & 1 & 1 & 50.00 & 0 & 0.00 & 1 & 50.0 \\
\hline Orthopedics & 45 & 39 & 6 & 13.33 & 5 & 11.11 & 1 & 2.22 \\
\hline Pediatrics & 911 & 836 & 75 & 8.23 & 65 & 7.14 & 10 & 1.10 \\
\hline Pediatric ICU & 1,239 & 1,122 & 117 & 9.44 & 98 & 7.91 & 19 & 1.53 \\
\hline Private ward & 84 & 79 & 5 & 5.95 & 4 & 4.76 & 1 & 1.19 \\
\hline Urology & 45 & 43 & 2 & 4.44 & 2 & 4.44 & 0 & 0.00 \\
\hline Total & 5,867 & 5,127 & 740 & 12.61 & 669 & 11.40 & 71 & 1.21 \\
\hline
\end{tabular}

Abbreviations: CTVS, cardiothoracic and vascular surgery; ICU, intensive care unit.

chances of recovery of isolates from peripheral venipuncture were observed to be significantly higher for Candida spp., Enterococcus (HLAR) spp., Salmonella typhi, S. aureus, S. pneumoniae, and other $\alpha$ and $\beta$ hemolytic Streptococcus spp. A description about the same attribute is depicted in - Table 5. The antibiotic susceptibility patterns for GPCs and GNBs were interpreted in accordance to prevalent CLSI guidelines ${ }^{6}$ and are represented in - Tables 6 and 7, respectively.

Beta lactams proved least effective for GPCs with sensitivity lowest in penicillin (29.6\%), ceftriaxone (45.6\%), and cephalexin (52.6\%). Erythromycin (31.7\%) and rifampicin (48.6\%) were largely ineffective. Relative sensitivity was highest for vancomycin (96.5\%) and linezolid (90.2\%). Even broad-spectrum antibiotics like chloramphenicol (78.5\%) and tetracycline (74.8\%) had good sensitivity against GPCs, especially MRSA. MSSA isolates uniformly showed very high sensitivities of $>85 \%$ to ampicillin, gentamicin, amikacin, tetracycline, chloramphenicol, levofloxacin, and cefotaxime. Streptococcus pneumoniae showed an incredibly good sensitivity to amoxicillin (100\%), cefepime (100\%), and penicillin (80\%).

Neisseria meningitidis isolates showed high sensitivity to penicillin (81.8\%), azithromycin (75\%), and ceftriaxone (66.67\%). Interestingly, ciprofloxacin (41.67\%) was only moderately effective. This is important because ciprofloxacin was the antimicrobial initially used to control the outbreak and also for the prophylaxis of contacts.

For GNBs, carbapenems were the most effective drugs (approximately $>85 \%$ sensitivity). Cefoperazone-sulbactam (CFS, $83.1 \%$ ) was the only other drug with sensitivity $\geq 80 \%$. Quinolones were much less effective. Injectables like gentamicin (58\%) and amikacin (63\%) showed moderate sensitivity. Cephalosporins were mostly ineffective with sensitivities as low as $10 \%$. K. pneumoniae and E. coli were highly susceptible to carbapenems ( $\geq 95 \%$ sensitivity) and CFS. E. coli additionally had better susceptibility ( $\geq 85 \%$ ) against aminoglycosides too. Quinolones were moderately effective for Klebsiella spp. (60-75\% sensitivity) but serve as very poor drugs to treat $E$. coli (15-20\% sensitivity) BSIs. A. baumannii was most sensitive to carbapenems, CFS, moderately sensitive to quinolones, gentamicin, piperacillin-tazobactam. However, Acinetobacter lwoffii documented better sensitivity to aminoglycosides and quinolones compared with carbapenems. P. aeruginosa isolated in $14.4 \%$ BSIs, was better managed with imipenem, CFS ( $>80 \%$ sensitivity), piperacillin-tazobactam, and levofloxacin, whereas typical antipseudomonal drugs like cefoperazone and ceftazidime were largely ineffective. Nonfermenting GNBs (other than Acinetobacter and Pseudomonas spp.) were highly susceptible to quinolones, meropenem, CFS, piperacillin-tazobactam, and chloramphenicol. Imipenem and gentamicin were drugs which were moderately sensitive 
Table 3 Day-wise distribution of blood stream pathogens recovered

\begin{tabular}{|c|c|c|c|c|c|c|c|c|}
\hline & Day 1 & Day 2 & Day 3 & Day 4 & Day 5 & Day 6 & Day 7 & Total \\
\hline \multicolumn{9}{|l|}{ Gram-positive cocci } \\
\hline Staphylococcus aureus (MSSA) & 5 & 20 & 13 & 12 & 4 & 7 & 6 & 67 \\
\hline Staphylococcus aureus (MRSA) & 4 & 7 & 17 & 13 & 9 & 8 & 3 & 61 \\
\hline CONS & 0 & 3 & 2 & 1 & 0 & 1 & 0 & 7 \\
\hline Enterococcus spp. & 3 & 9 & 4 & 0 & 0 & 3 & 1 & 20 \\
\hline Enterococcus spp. (HLAR) & 2 & 4 & 0 & 1 & 1 & 0 & 2 & 10 \\
\hline$\alpha$-hemolytic Streptococcus spp. & 6 & 4 & 3 & 4 & 0 & 1 & 0 & 18 \\
\hline$\beta$-hemolytic Streptococcus spp. & 0 & 2 & 2 & 0 & 0 & 0 & 1 & 5 \\
\hline Streptococcus pneumoniae & 0 & 4 & 1 & 2 & 0 & 0 & 0 & 7 \\
\hline Total & & & & & & & & $n=195$ \\
\hline \multicolumn{9}{|l|}{ Gram-negative bacilli } \\
\hline Escherichia coli & 11 & 17 & 4 & 1 & 1 & 1 & 0 & 35 \\
\hline Klebsiella oxytoca & 2 & 2 & 1 & 0 & 0 & 0 & 0 & 5 \\
\hline Klebsiella pneumoniae & 29 & 63 & 13 & 5 & 9 & 1 & 5 & 125 \\
\hline Nonfermenter GNB & 3 & 11 & 1 & 6 & 6 & 4 & 4 & 35 \\
\hline Acinetobacter baumannii & 14 & 39 & 12 & 12 & 9 & 2 & 10 & 98 \\
\hline Acinetobacter Iwoffii & 1 & 8 & 3 & 0 & 5 & 1 & 4 & 22 \\
\hline Proteus spp. & 0 & 0 & 2 & 0 & 0 & 1 & 0 & 3 \\
\hline Proteus mirabilis & 0 & 2 & 0 & 0 & 0 & 0 & 0 & 2 \\
\hline Morganella morganii & 1 & 1 & 0 & 0 & 0 & 0 & 0 & 2 \\
\hline Enterobacter spp. & 3 & 10 & 2 & 0 & 1 & 5 & 4 & 25 \\
\hline Citrobacter freundii & 0 & 2 & 1 & 0 & 0 & 0 & 0 & 3 \\
\hline Citrobacter diversus & 0 & 2 & 1 & 0 & 0 & 1 & 0 & 4 \\
\hline Pseudomonas spp. & 0 & 0 & 0 & 0 & 1 & 0 & 1 & 2 \\
\hline Pseudomonas aeruginosa & 3 & 26 & 16 & 10 & 1 & 4 & 3 & 63 \\
\hline Salmonella paratyphi & 0 & 0 & 4 & 0 & 0 & 0 & 0 & 4 \\
\hline Salmonella enteritidis & 1 & 0 & 0 & 0 & 0 & 0 & 0 & 1 \\
\hline Salmonella typhi & 0 & 2 & 1 & 0 & 0 & 1 & 2 & 6 \\
\hline Hemophilus influenzae & 0 & 1 & 0 & 0 & 0 & 0 & 0 & 1 \\
\hline Total & & & & & & & & $n=436$ \\
\hline \multicolumn{9}{|l|}{ Others } \\
\hline Neisseria meningitidis & 1 & 2 & 8 & 0 & 1 & 1 & 2 & 15 \\
\hline Listeria monocytogenes & 0 & 0 & 0 & 0 & 1 & 0 & 0 & 1 \\
\hline Candida spp. & 0 & 1 & 4 & 0 & 4 & 2 & 0 & 11 \\
\hline GRAND TOTAL & 89 & 242 & 115 & 67 & 53 & 44 & 48 & 658 \\
\hline
\end{tabular}

Abbreviations: CoNS, coagulase negative Staphylococcus spp.; GNB, gram-negative bacilli; HLAR, high-level aminoglycoside resistance; MRSA, methicillin-resistant Staphylococcus aureus; MSSA, methicillin-sensitive Staphylococcus aureus.

to these organisms. Morganella, Salmonella, and Hemophilus spp. isolates recovered were completely susceptible to usual drugs tested and no major antimicrobial resistance trends were observed in them.

\section{Discussion}

BSI constitutes one of the major causes of morbidity and mortality. Definitive diagnosis is established by bacteriologic culture of blood samples to identify organisms and provide antimicrobial susceptibility. ${ }^{10}$ Numerous analyses have concluded that early treatment of bacteremic patients with an appropriate antimicrobial drug improves survival. ${ }^{11-14}$ There exists a strong relationship between delay in effective initiation of therapy and in-hospital mortality of septic shock. Each hour of delay in therapy initiation is associated with an average decrease in survival of $8 \% .{ }^{15}$ Defining pathogen distribution and drug resistance patterns provides basis for empirical as well as definitive therapy. ${ }^{16}$ This present study is an attempt to analyze the bacterial 
Table 4 Comparison of yield of pathogens after aerobic incubation between 48 h vs. $7 \mathrm{~d}$

\begin{tabular}{|c|c|c|c|c|c|c|c|}
\hline & \multicolumn{4}{|c|}{ Day } & \multirow[t]{3}{*}{ Total } & \multirow{2}{*}{\multicolumn{2}{|c|}{ Significance test }} \\
\hline & \multicolumn{2}{|r|}{$48 \mathrm{~h}$} & \multicolumn{2}{|r|}{$7 d$} & & & \\
\hline & $N$ & $\%$ & $N$ & $\%$ & & Z-Stat & $p$-Value \\
\hline Acinetobacter baumannii & 53 & $16.01 \%$ & 45 & $13.76 \%$ & 98 & 0.81 & 0.417 \\
\hline Acinetobacter Iwoffii & 9 & $2.72 \%$ & 13 & $3.98 \%$ & 22 & -0.9 & 0.37 \\
\hline Candida spp. & 1 & $0.30 \%$ & 10 & $3.06 \%$ & 11 & -2.76 & 0.006 \\
\hline Citrobacter spp. & 4 & $1.21 \%$ & 3 & $0.92 \%$ & 7 & 0.36 & 0.716 \\
\hline CONS & 3 & $0.91 \%$ & 4 & $1.22 \%$ & 7 & -0.4 & 0.692 \\
\hline Enterobacter spp. & 13 & $3.93 \%$ & 12 & $3.67 \%$ & 25 & 0.17 & 0.863 \\
\hline Enterococcus spp. & 12 & $3.63 \%$ & 8 & $2.45 \%$ & 20 & 0.88 & 0.378 \\
\hline Enterococcus spp. (HLAR) & 6 & $1.81 \%$ & 4 & $1.22 \%$ & 10 & 0.62 & 0.536 \\
\hline Escherichia coli & 28 & $8.46 \%$ & 7 & $2.14 \%$ & 35 & 3.66 & $<0.001$ \\
\hline Hemophilus influenzae & 1 & $0.30 \%$ & 0 & $0.00 \%$ & 1 & -1 & 0.317 \\
\hline Klebsiella oxytoca & 4 & $1.21 \%$ & 1 & $0.31 \%$ & 5 & 1.34 & 0.18 \\
\hline Klebsiella pneumoniae & 92 & $27.79 \%$ & 33 & $10.09 \%$ & 125 & 5.95 & $<0.001$ \\
\hline Listeria monocytogenes & 0 & $0.00 \%$ & 1 & $0.31 \%$ & 1 & -1 & 0.317 \\
\hline Morganella morganii & 2 & $0.60 \%$ & 0 & $0.00 \%$ & 2 & 1.42 & 0.156 \\
\hline Neisseria meningitidis & 3 & $0.91 \%$ & 12 & $3.67 \%$ & 15 & -2.38 & 0.017 \\
\hline Nonfermenter GNB & 14 & $4.23 \%$ & 21 & $6.42 \%$ & 35 & -1.25 & 0.21 \\
\hline Proteus spp. & 2 & $0.60 \%$ & 3 & $0.92 \%$ & 5 & -0.46 & 0.644 \\
\hline Pseudomonas aeruginosa & 29 & $8.76 \%$ & 34 & $10.40 \%$ & 63 & -0.71 & 0.476 \\
\hline Pseudomonas spp. & 0 & $0.00 \%$ & 2 & $0.61 \%$ & 2 & -1.42 & 0.156 \\
\hline Salmonella enteritidis & 1 & $0.30 \%$ & 0 & $0.00 \%$ & 1 & 1 & 0.317 \\
\hline Salmonella paratyphi & 0 & $0.00 \%$ & 4 & $1.22 \%$ & 4 & -2.01 & 0.044 \\
\hline Salmonella Typhi & 2 & $0.60 \%$ & 4 & $1.22 \%$ & 6 & -0.83 & 0.404 \\
\hline Staphylococcus aureus (MRSA) & 11 & $3.32 \%$ & 50 & $15.29 \%$ & 61 & -5.39 & $<0.001$ \\
\hline Staphylococcus aureus (MSSA) & 25 & $7.55 \%$ & 42 & $12.84 \%$ & 67 & -2.25 & 0.024 \\
\hline Streptococcus pneumoniae & 4 & $1.21 \%$ & 3 & $0.92 \%$ & 7 & 0.36 & 0.716 \\
\hline$\alpha$-hemolytic Streptococcus spp. & 10 & $3.02 \%$ & 8 & $2.45 \%$ & 18 & 0.45 & 0.651 \\
\hline$\beta$-hemolytic Streptococcus spp. & 2 & $0.60 \%$ & 3 & $0.92 \%$ & 5 & -0.46 & 0.644 \\
\hline Total & 331 & $100.00 \%$ & 327 & $100.00 \%$ & 658 & & \\
\hline
\end{tabular}

Abbreviations: CoNS, coagulase negative Staphylococcus spp.; GNB, gram-negative Bacilli; HLAR, high-level aminoglycoside resistance; MRSA, methicillin-resistant Staphylococcus aureus; MSSA, methicillin-sensitive Staphylococcus aureus.

profile of blood culture isolates, assess antimicrobial trends, correlate bacteremic source and their impact, and discuss other variables which may help us devise the best ways of managing BSIs.

This is a 5-year retrospective analysis of 5,867 blood samples received from clinically suspected bacteremia patients. A total of 658 recognized pathogens were recovered from 637 specimens. A total of $97 \%$ isolates had monomicrobial growths. The rate of isolation for blood culture pathogens was observed at $11.2 \%$. This is significant considering it is a new tertiary level 450-bedded hospital apart from the fact that the laboratory uses conventional blood culture bottles with no provision for continuous monitoring of culture bottles. Besides, this rate of isolation is in consonance with many studies from India ${ }^{17-22}$ and abroad. ${ }^{23-25}$
Although the mean age of bacteremic patients in the present study was 33.7 years, the highest number of cases of sepsis and shock were observed in children younger than 5 years age group as well as adults from 21 to 40 years age bracket. After the age of 40 years, the incidence of bacteremia cases shows a steady decline with every passing decade. Neonates, infants, and young children are particularly vulnerable to BSI owing to numerous risk factors like premature rupture of membrane, prolonged rupture, prematurity, recurrent urinary tract infection, poor maternal nutrition, low birthweight, birth asphyxia, congenital anomalies, and nascent/ weak host immunity. ${ }^{10}$

Almost $31 \%$ of all medical ICU patients suspected with bacteremia were confirmed by culture. This is understood as patients admitted in ICU are usually already severely ill who are put under continued and enhanced vigilant care. Since 
Table 5 Comparative data about blood stream infections from central line vs. peripheral lines ${ }^{a}$

\begin{tabular}{|c|c|c|c|c|c|c|c|}
\hline \multirow[b]{4}{*}{ Acinetobacter baumannii } & \multicolumn{4}{|c|}{ Line } & \multirow[t]{3}{*}{ Total } & \multicolumn{2}{|c|}{ Significance test } \\
\hline & Central & $\%$ & Peripheral & $\%$ & & & \\
\hline & \multicolumn{2}{|l|}{$n=93$} & \multicolumn{2}{|l|}{$n=395$} & & Z-Stat & $p$-Value \\
\hline & 26 & $27.96 \%$ & 59 & $14.94 \%$ & 85 & 2.6100 & 0.0090 \\
\hline Acinetobacter Iwoffii & 3 & $3.23 \%$ & 12 & $3.04 \%$ & 15 & 0.0900 & 0.9260 \\
\hline Candida spp. & 0 & $0.00 \%$ & 5 & $1.27 \%$ & 5 & -2.2500 & 0.0240 \\
\hline Citrobacter spp. & 0 & $0.00 \%$ & 2 & $0.51 \%$ & 2 & -1.4200 & 0.1560 \\
\hline CONS & 1 & $1.08 \%$ & 3 & $0.76 \%$ & 4 & 0.2700 & 0.7850 \\
\hline Enterobacter spp. & 3 & $3.23 \%$ & 16 & $4.05 \%$ & 19 & -0.4000 & 0.692 \\
\hline Enterococcus spp. & 3 & $3.23 \%$ & 12 & $3.04 \%$ & 15 & 0.0900 & 0.9260 \\
\hline Enterococcus spp. (HLAR) & 0 & $0.00 \%$ & 6 & $1.52 \%$ & 6 & -2.4700 & 0.0140 \\
\hline Escherichia coli & 3 & $3.23 \%$ & 23 & $5.82 \%$ & 26 & -1.1900 & 0.2330 \\
\hline Hemophilus influenzae & 0 & $0.00 \%$ & 1 & $0.25 \%$ & 1 & -1.0000 & 0.3170 \\
\hline Klebsiella oxytoca & 1 & $1.08 \%$ & 2 & $0.51 \%$ & 3 & 0.5000 & 0.4700 \\
\hline Klebsiella pneumoniae & 31 & $33.33 \%$ & 68 & $17.22 \%$ & 99 & 3.0700 & 0.0020 \\
\hline Listeria monocytogenes & 0 & $0.00 \%$ & 1 & $0.25 \%$ & 1 & -1.0000 & 0.3170 \\
\hline Morganella morganii & 1 & $1.08 \%$ & 1 & $0.25 \%$ & 2 & 0.7500 & 0.4540 \\
\hline Neisseria meningitidis & 0 & $0.00 \%$ & 1 & $0.25 \%$ & 1 & -1.0000 & 0.3170 \\
\hline $\begin{array}{l}\text { Nonfermenting } \\
\text { gram-negative Bacilli }\end{array}$ & 4 & $4.30 \%$ & 29 & $7.34 \%$ & 33 & -1.2300 & 0.2200 \\
\hline Proteus spp. & 2 & $2.15 \%$ & 3 & $0.76 \%$ & 5 & 0.8900 & 0.3750 \\
\hline Pseudomonas aeruginosa & 11 & $11.83 \%$ & 39 & $9.87 \%$ & 50 & 0.5300 & 0.5940 \\
\hline Pseudomonas spp. & 0 & $0.00 \%$ & 0 & $0.00 \%$ & 0 & $\mathrm{NA}$ & \\
\hline Salmonella enteritidis & 0 & $0.00 \%$ & 1 & $0.25 \%$ & 1 & -1.0000 & 0.3170 \\
\hline Salmonella paratyphi & 0 & $0.00 \%$ & 3 & $0.76 \%$ & 3 & -1.7400 & 0.0820 \\
\hline Salmonella typhi & 0 & $0.00 \%$ & 4 & $1.01 \%$ & 4 & -2.0100 & 0.0440 \\
\hline $\begin{array}{l}\text { Staphylococcus aureus } \\
\text { (MRSA) }\end{array}$ & 2 & $2.15 \%$ & 38 & $9.62 \%$ & 40 & -3.5400 & $<0.001$ \\
\hline $\begin{array}{l}\text { Staphylococcus aureus } \\
\text { (MSSA) }\end{array}$ & 2 & $2.15 \%$ & 45 & $11.39 \%$ & 47 & -4.2100 & $<0.001$ \\
\hline $\begin{array}{l}\text { Streptococcus } \\
\text { pneumoniae }\end{array}$ & 0 & $0.00 \%$ & 5 & $1.27 \%$ & 5 & -2.2500 & 0.0240 \\
\hline $\begin{array}{l}\alpha \text {-Hemolytic } \\
\text { Streptococcus spp. }\end{array}$ & 0 & $0.00 \%$ & 11 & $2.78 \%$ & 11 & -3.3600 & 0.0010 \\
\hline \multirow[t]{2}{*}{$\begin{array}{l}\beta \text {-Hemolytic } \\
\text { Streptococcus spp. }\end{array}$} & 0 & $0.00 \%$ & 5 & $1.27 \%$ & 5 & -2.2500 & 0.0240 \\
\hline & 93 & $19.02 \%$ & 395 & $80.78 \%$ & 488 & & \\
\hline
\end{tabular}

Abbreviations: CoNS, coagulase negative Staphylococcus spp.; HLAR, high-level aminoglycoside resistance; MRSA, methicillin-resistant Staphylococcus aureus; MSSA, methicillin-sensitive Staphylococcus aureus.

aData for 2011 to 2013 only.

they are better equipped to handle such serious infections, critical patients are referred to ICUs more frequently from other departments which explains the highest positivity yield of blood culture specimens received from medical ICU.

The rate of contamination observed (1.21\%) is below the target level suggested by Hall and Lyman. ${ }^{7}$ This correlates well with other studies by Archibald et $\mathrm{al}^{26}$ and Weinstein. ${ }^{9}$ These included mainly isolates of Bacillus spp. Corynebacterium spp., and Micrococcus spp.

Among 658 pathogens isolated, GNB were significantly the predominant organisms. This corresponds to findings documented by other similar studies. ${ }^{10,17,21}$ Klebsiella spp. as a dominant microbe causing BSIs was also reported by Roy et al $^{21}$ and Tariq. ${ }^{23}$

Within GPCs, S. aureus (65.6\%) 'was the predominant pathogenic organism isolated.' Pre-eminence of $S$. aureus as a bloodstream pathogen has been documented by numerous similar studies. ${ }^{10,18,25,27}$ If a comparison of methicillin sensitivity is attempted among S. aureus strains, 52.34\% were MSSA and rest were MRSA. MRSA are notorious since these are resistant to action of a broad group of $\beta$-lactam antibiotics, which cannot be used for therapy. One-third (10/30) of Enterococcus 
Table 6 Drug sensitivity profile of gram-positive strains

\begin{tabular}{|c|c|c|c|c|c|c|c|c|c|}
\hline Drug & $\begin{array}{l}\text { Overall } \\
\text { GPC }\end{array}$ & $\begin{array}{l}\text { Enterococcus } \\
\text { spp. }\end{array}$ & $\begin{array}{l}\text { Enterococcus } \\
\text { spp. (HLAR) }\end{array}$ & MRSA & MSSA & $\begin{array}{l}\text { Streptococcus } \\
\text { pneumoniae }\end{array}$ & CoNS & $\begin{array}{l}\alpha \text {-Hemolytic } \\
\text { Streptococcus } \\
\text { spp. }\end{array}$ & $\begin{array}{l}\text { Neisseria } \\
\text { meningitidis }\end{array}$ \\
\hline Imipenem & 85.7 & 90.9 & 60.0 & & & & & & \\
\hline Ciprofloxacin & 57.3 & 58.3 & 0.0 & 39.6 & 76.3 & & 50.0 & 100.0 & 41.7 \\
\hline Penicillin & 29.6 & 35.7 & 100.0 & 10.3 & 100.0 & 80.0 & 33.3 & 77.8 & 81.8 \\
\hline Ampicillin & 71.4 & 68.4 & 50.0 & & 100.0 & & & 81.8 & \\
\hline Vancomycin & 96.5 & 100.0 & 87.5 & 96.3 & & 100.0 & 100.0 & 100.0 & \\
\hline Linezolid & 90.2 & 90.0 & 100.0 & 96.3 & & 100.0 & 66.7 & & \\
\hline Gentamicin & 79.8 & 86.7 & 0.0 & 65.3 & 97.9 & & 80.0 & 80.0 & \\
\hline Tetracycline & 74.8 & 42.9 & 20.0 & 83.3 & 87.2 & 50.0 & 25.0 & 100.0 & \\
\hline Amikacin & 68.9 & 0.0 & 0.0 & 85.7 & 100.0 & & & & \\
\hline Levofloxacin & & 33.3 & 0.0 & 60.0 & 91.3 & 50.0 & 100.0 & & \\
\hline Cefotaxime & 67.8 & 100.0 & 0.0 & 23.1 & 90.0 & 75.0 & 83.3 & 83.3 & \\
\hline Chloramphenicol & 78.5 & & 50.0 & 70.6 & 88.5 & 60.0 & 50.0 & 100.0 & 50.0 \\
\hline Ofloxacin & 61.4 & & & 46.8 & 86.1 & & 0.0 & & \\
\hline Netilmicin & 85.9 & & & 81.3 & 97.1 & & & & \\
\hline Rifampicin & 48.6 & & & 33.3 & & 0.0 & 0.0 & 100.0 & 50.0 \\
\hline Cephalexin & 52.6 & & & 10.5 & 100.0 & & & & \\
\hline Clindamycin & & & & 50.0 & & & & & \\
\hline Erythromycin & 31.7 & & & 7.9 & 47.1 & & 40.0 & 100.0 & \\
\hline Ceftriaxone & 45.3 & & & 9.7 & 73.5 & 50.0 & 50.0 & & 66.7 \\
\hline Cefepime & & & & 0.0 & 75.0 & 100.0 & & & \\
\hline Azithromycin & & & & & & & & & 75.0 \\
\hline Amoxicillin & & & & & & 100.0 & 100.0 & & \\
\hline Norfloxacin & & & & & & & 100.0 & & \\
\hline
\end{tabular}

Abbreviations: CoNS, coagulase negative Staphylococcus spp.; GPC, gram-positive Cocci; HLAR, high-level aminoglycoside resistance; MRSA, methicillin-resistant Staphylococcus aureus; MSSA, methicillin-sensitive Staphylococcus aureus.

spp. isolates carried genes for HLAR, where a combination of $\beta$-lactams and aminoglycosides may not work in vivo for therapy even though they may have been sensitive in vitro. Coagulase negative Staphylococcus (CoNS), long considered as contaminants in 1970s and 1980s are nowadays considered to be agents capable of causing bacteremia. They are mostly incriminated as nosocomial pathogens specifically in catheter-related BSI. In fact, two studies ${ }^{17,28}$ reported CoNS as the most common isolate causing BSIs in ICU patients. Seven such isolates of CoNS were recovered in the present study which fulfilled the criteria for sepsis.

All patients with fungemia were critical and under observation in different ICUs. Risk factors for fungemia include prolonged hospital stay, hyper alimentation, previous broad-spectrum antimicrobial therapy, and ulcerations in gastrointestinal mucosa. Prognosis in fungemia patients is relatively poor. ${ }^{29}$

Frequency of isolation on different days of incubation within the 7-day incubation period reveals interesting trends.
Most of the prominent isolates had a peak of detection on the second day of incubation which gradually tapered within the next 2 days. However, there was no significant difference observed between organisms isolated within the first 48 hours or beyond initial 48 hours.

An attempt was made to evaluate any correlation between the source of blood collection and the frequency of organisms isolated. The data for this information were available roughly for approximately 3 years (2011-2013) only. Chances of recovery of positive blood culture isolates from central line catheters were significantly higher in A. baumannii $(p<0.0029)$ and K. pneumoniae $(p<0.002)$. This is easily comprehensible since central venous catheters are kept in situ for longer durations compared with peripherally inserted catheters. These organisms are known to produce biofilm which improves their persistence within intravascular devices. Biofilm formation also imparts them the ability to reduce their metabolism rate, prevent antibiotic entry, and promote transfer of resistance plasmids. 


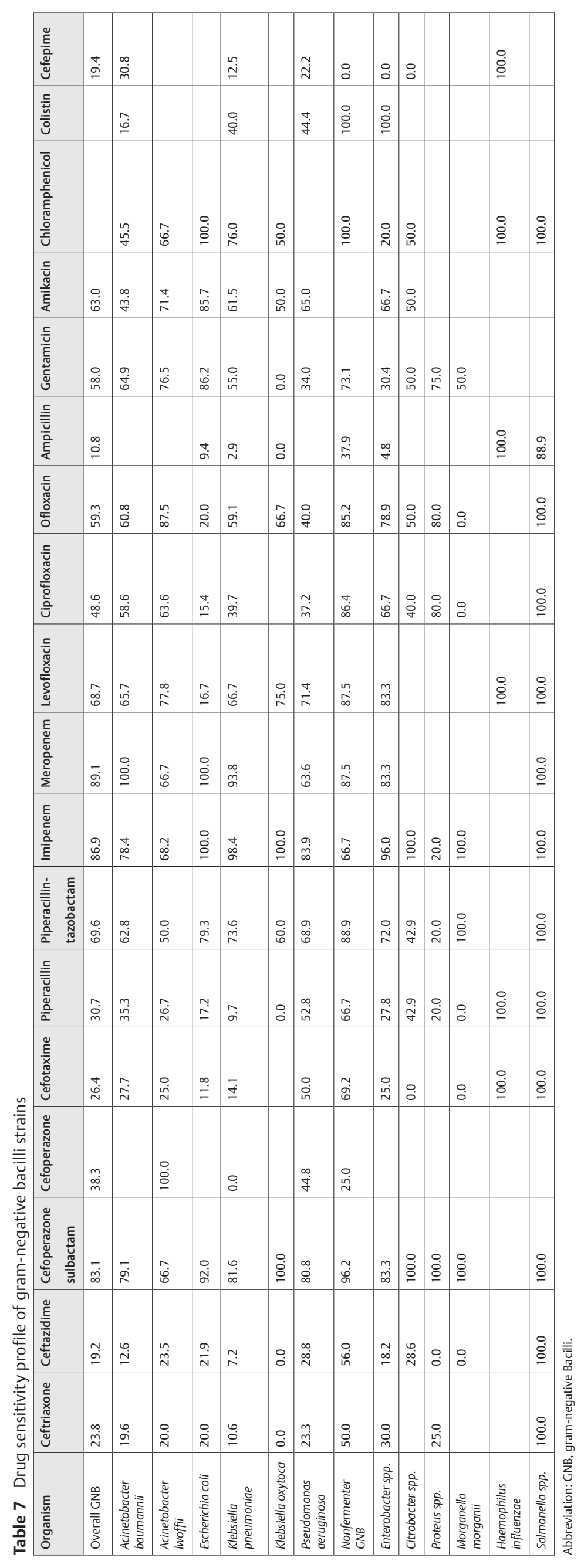


Such foci of bacteria can lead to sustained bacteremia if not dislodged in time, or catheter being removed. Contamination rates were similar for both sources of blood collection at approximately $1 \%$. A study by Beutz et $\mathrm{al}^{30}$ claims sensitivity of 92.5 and $95.9 \%$ for blood cultures drawn from central vein catheters and peripheral venipuncture, respectively. Even though the negative predictive values of both sources of blood collection was $>95 \%$, positive predictive values of blood cultures were low at 58.3 and $66.7 \%$, respectively.

Beta lactam drugs are rapidly becoming ineffective for treating BSIs because of its indiscriminate and nonjudicious usage. Vancomycin, linezolid, aminoglycosides, and broad-spectrum drugs like chloramphenicol and tetracycline are the most reliable treatment options for GPCs, whereas carbapenems, CFS, aminoglycosides, and quinolones are remaining treatment options for GNBs. Aminoglycosides are good options for E. coli but not K. pneumoniae BSIs, while the opposite is true for quinolones. Cephalosporins and typical antipseudomonal drugs failed terribly in controlling concerned BSIs. Hence rationalized drug therapy is the call of the hour and therefore, studies of this type are quite warranted. De-escalation of high-end antimicrobials once actual sensitivity pattern is known contributes to reduction of antimicrobial pressure. Poor infection control practices and inappropriate use of antibiotics are main driving forces for the spread of resistant organisms. Aggressive measures like routine surveillance cultures to identify and isolate carriers, control of environmental sources, antibiotic restriction, antibiotic recycling, recommending combination therapy, implementing proper aseptic techniques, performing hand hygiene, maintaining robust infection control practices, and periodical assessment of antimicrobial policy will go a long way in preventing emergence of resistant organisms.

\section{Conclusion}

Successful treatment of sepsis cases hinges on early diagnosis and proper antimicrobial therapy. The choice of antibiotics is based upon local knowledge of bacteriological profile and antimicrobial sensitivity patterns. The serious nature of the BSIs underscores the importance of periodic epidemiological surveillance studies such as the current one to provide useful insights for rational policy development and management of similar infections.

\section{Financial Support}

None.

\section{Ethical Approval \\ Approved.}

\section{Informed Consent}

Retrospective observational analysis, consent could not be obtained.

\section{Conflict of Interest}

None declared.

\section{Acknowledgment}

The authors would like to thank Mr. Pandurang V. Thatkar for technical and statistical help.

\section{References}

1 Seifert $\mathrm{H}$, Wisplinghoff $\mathrm{H}$, Bloodstream infection and endocarditis. In: Borriello SP, Murray PR, Funke G, eds. Topley and Wilson's Microbiology and Microbial Infections, Bacteriology Vol 1. 10th ed. London: Hodder Arnold ASM Press; 2005 1181-1235

2 Munford RS, Suffredini AF, Sepsis, severe sepsis and septic shock. In: Bennett JE, Dolin R, Blaser MJ, eds. Mandell, Douglas and Bennett's Principles and Practice of Infectious Diseases Vol 1. 8th ed. Philadelphia, PA: Elsevier Saunders; 2015 914-934

3 Vasudeva N, Nirwan PS, Shrivastava P. Bloodstream infections and antimicrobial sensitivity patterns in a tertiary care hospital of India. Ther Adv Infect Dis 2016;3(5):119-127

4 Weinstein MP. Current blood culture methods and systems: clinical concepts, technology, and interpretation of results. Clin Infect Dis 1996;23(1):40-46

5 CLSI, Principles and Procedures for Blood Cultures; Approved Guideline. CLSI Document M47-A.Wayne, PA: Clinical and Laboratory Standards Institute; 2007

6 Performance standards for Antimicrobial Susceptibility Testing, Twenty-Fourth Informational Supplement. CLSI Document M100-S24. Wayne, PA: Clinical and Laboratory Standards Institute; 2014

7 Hall KK, Lyman JA. Updated review of blood culture contamination. Clin Microbiol Rev 2006;19(4):788-802

8 Weinstein MP, Reller LB, Murphy JR, Lichtenstein KA. The clinical significance of positive blood cultures: a comprehensive analysis of 500 episodes of bacteremia and fungemia in adults. I. Laboratory and epidemiologic observations. Rev Infect Dis 1983;5(1):35-53

9 Weinstein MP. Blood culture contamination: persisting problems and partial progress. J Clin Microbiol 2003;41(6): 2275-2278

10 Prabhu K, Bhat S, Rao S. Bacteriologic profile and antibiogram of blood culture isolates in a pediatric care unit. J Lab Physicians 2010;2(2):85-88

11 Kollef MH, Sherman G, Ward S, Fraser VJ. Inadequate antimicrobial treatment of infections: a risk factor for hospital mortality among critically ill patients. Chest 1999;115(2): 462-474

12 Leibovici L, Paul M, Poznanski O, et al. Monotherapy versus beta-lactam-aminoglycoside combination treatment for gram-negative bacteremia: a prospective, observational study. Antimicrob Agents Chemother 1997;41(5):1127-1133

13 Leibovici L, Shraga I, Drucker M, Konigsberger H, Samra Z, Pitlik SD. The benefit of appropriate empirical antibiotic treatment in patients with bloodstream infection. J Intern Med 1998;244(5):379-386

14 Ibrahim EH, Sherman G, Ward S, Fraser VJ, Kollef MH. The influence of inadequate antimicrobial treatment of bloodstream infections on patient outcomes in the ICU setting. Chest 2000;118(1):146-155

15 Kumar A, Roberts D, Wood KE, et al. Duration of hypotension before initiation of effective antimicrobial therapy is the critical determinant of survival in human septic shock. Crit Care Med 2006;34(6):1589-1596

16 Jagdish L, Naik TB, Gupta RK, Jais M. Etiology of blood culture from septicemia cases and their antibiotic susceptibility pattern at a tertiary care hospital. Indian J Microbiol Res 2016;3:436-440

17 Wattal C, Raveendran R, Goel N, Oberoi JK, Rao BK. Ecology of blood stream infection and antibiotic resistance in intensive 
care unit at a tertiary care hospital in North India. Braz J Infect Dis 2014;18(3):245-251

18 Rajeevan S, Ahmed SM, Jasmin PT. Study of prevalence and antimicrobial susceptibility pattern in blood isolates from a tertiary care hospital in North Kerala, India. Int J Curr Microbiol Appl Sci 2014;3:655-662

19 Sharma R, Sharma R, Gupta S. Bacteriological analysis of blood culture isolates with their antibiogram from a tertiary care hospital. Int J Pharm Sci Res 2015;6:4847-4851

20 Gupta S, Kashyap B. Bacteriological profile and antibiogram of blood culture isolates from a tertiary care hospital of North India. Trop J Med Res 2016;19:94-99

21 Roy I, Jain A, Kumar M, Agarwal SK. Bacteriology of neonatal septicaemia in a tertiary care hospital of northern India. Indian J Med Microbiol 2002;20(3):156-159

22 Gohel K, Jojera A, Soni S, Gang S, Sabnis R, Desai M. Bacteriological profile and drug resistance patterns of blood culture isolates in a tertiary care nephrourology teaching institute. BioMed Res Int 2014;2014:153747

23 Tariq TM. Bacteriologic profile and antibiogram of blood culture isolates from a children's hospital in Kabul. J Coll Physicians Surg Pak 2014;24(6):396-399

24 Quereshi M, Aziz F. Prevalence of microbial isolates in blood cultures and their antimicrobial susceptibility profiles. Biomedica 2011;27:136-139
25 Meremikwu MM, Nwachukwu CE, Asuquo AE, Okebe JU, Utsalo SJ. Bacterial isolates from blood cultures of children with suspected septicaemia in Calabar, Nigeria. BMC Infect Dis 2005;5:110

26 Archibald LK, Pallangyo K, Kazembe P, Reller LB. Blood culture contamination in Tanzania, Malawi, and the United States: a microbiological tale of three cities. J Clin Microbiol 2006;44(12):4425-4429

27 Diekema DJ, Beekmann SE, Chapin KC, Morel KA, Munson E, Doern GV. Epidemiology and outcome of nosocomial and community-onset bloodstream infection. J Clin Microbiol 2003;41(8):3655-3660

28 Karlowsky JA, Jones ME, Draghi DC, Thornsberry C, Sahm DF, Volturo GA. Prevalence and antimicrobial susceptibilities of bacteria isolated from blood cultures of hospitalized patients in the United States in 2002. Ann Clin Microbiol Antimicrob 2004;3:7

29 Bryan CS. Clinical implications of positive blood cultures. Clin Microbiol Rev 1989;2(4):329-353

30 Beutz M, Sherman G, Mayfield J, Fraser VJ, Kollef MH. Clinical utility of blood cultures drawn from central vein catheters and peripheral venipuncture in critically ill medical patients. Chest 2003;123(3):854-861 